\title{
Adaptive Generalized Space Shift Keying (GSSK) Modulation for MISO Channels: A New Method for High Diversity and Coding Gains
}

\author{
Konstantinos Ntontin ${ }^{(1)}$, Marco Di Renzo ${ }^{(2)}$, Ana Perez-Neira ${ }^{(1)}$, and Christos Verikoukis ${ }^{(1)}$ \\ ${ }^{(1)}$ Telecommunications Technological Centre of Catalonia (CTTC) \\ Castelldefels, Spain \\ Email: constantinos.ntontin@cttc.es, anuska@gps.tsc.upc.edu, cveri@cttc.es \\ ${ }^{(2)}$ L2S, UMR 8506 CNRS - SUPELEC - Univ Paris-Sud, Laboratory of Signals and Systems (L2S) \\ French National Center for Scientific Research (CNRS), École Supérieure d'Électricité (SUPÉLEC) \\ University of Paris-Sud XI (UPS), 3 rue Joliot-Curie, 91192 Gif-sur-Yvette (Paris), France \\ Email: marco.direnzo@1ss.supelec.fr
}

\begin{abstract}
Generalized Space Shift Keying (GSSK) modulation is a recently proposed low-complexity concept for Multiple-InputMultiple-Output (MIMO) wireless systems. GSSK modulation is a generalized version of Space Shift Keying (SSK) modulation, which provides a better spectral efficiency through multiple active antennas at the transmitter. An apparent weakness of GSSK modulation is that it does not exploit the transmit-antennas to achieve transmit-diversity. In this paper, we propose a precoding method for GSSK modulation, which simultaneously achieves high diversity and coding gains. The solution is based on: i) cophasing the active antennas of each spatial-constellation point; and ii) properly rotating the phases among spatial-constellation points. The new scheme requires Channel State Information at the Transmitter (CSIT), i.e., the channel phases of each wireless link, which can be obtained through a feedback channel. For the case of a perfect feedback channel, we analytically show that for three and four antennas at the transmitter a full transmit diversity can be achieved without reducing the achievable rate. Furthermore, for various MISO configurations and achievable rates we show through Monte Carlo simulations that our proposed scheme outperforms state-of-the-art open-loop GSSK schemes, in terms of both diversity and coding gain, when the number of bits allocated for the quantization of each channel phase is between 2 and 4 .
\end{abstract}

\section{INTRODUCTION}

Spatial Modulation (SM) is a recently proposed MultipleInput-Multiple-Output (MIMO) technique, which allows information to be conveyed in two ways: i) through a spatial constellation diagram; and ii) through a signal-constellation diagram [1], [2]. Space Shift Keying (SSK) modulation is a lowcomplexity implementation of SM [3], which relies only on the spatial-constellation diagram to convey information. This way, the decoding process at the receiver becomes simpler at the cost of a reduced spectral efficiency. To compensate for this latter disadvantage, Generalized Space Shift Keying (GSSK) modulation has been recently proposed [4]. Unlike SM and SSK modulation, GSSK modulation avoids the restriction of having only one active transmit-antenna in each time-slot, and allows multiple antennas to be simultaneously active. As a result, it guarantees a better usage of the spatial degrees of freedom, by providing a trade-off between complexity and spectral efficiency.

However, GSSK modulation has two main limitations [5], [6]: i) energy is wasted by having common antennas in different spatial-constellation points; and ii) no transmit-diversity gain is achieved, even though multiple antennas are available at the transmitter. It has been shown in [4] that the achievable diversity order of GSSK modulation is equal to the number of antennas at the receiver, regardless of the number of active transmit-antennas.

Prior Work: To overcome these limitations, some methods have been recently proposed, which provide transmit-diversity for SM, SSK, and GSSK modulation. In [5], the authors have proposed the so-called Time Orthogonal Signal Design (TOSD) method for SSK modulation. The solution provides, regardless of the number of transmit-antennas, a transmitdiversity that is twice the number of antennas at the receiver. Space-Time Block Codes (STBCs) technology [17] has been proposed in [7] and [8] for SM, and it has been shown that a transmit-diversity equal to two can be achieved. A dispersion matrix approach has been proposed in [9], which exploits the temporal dimension to achieve transmit-diversity, and, thus, introduces a fundamental trade-off between rate and diversity. In [6], the authors have applied the TOSD framework to GSSK modulation, and have shown that the transmitdiversity order of the proposed TOSD-GSSK method is equal to the minimum number of distinct antenna elements among all the different pairs of spatial-constellation points. Consequently, even though transmit-diversity is achieved, energy inefficiency caused by having common antennas in different spatial constellation points is still present, along with a tradeoff between achievable rate and diversity. The aforementioned transmit-diversity methods for SM, SSK, and GSSK modulation are inherently open-loop, i.e., they do not require CSIT to achieve transmit-diversity. However, it is known that precoding methods that exploit CSIT can significantly improve the performance of MIMO systems, both in terms of diversity 
and coding gain [10]. As far as SM is concerned, [11], [12], and [14] have recently proposed precoding aided schemes to achieve better performance. In [11] and [14], solutions that exploit average CSIT, but do not achieve transmit-diversity are proposed. In [12], a time-slot phase feedback method for SSK systems with two transmit and one receive antenna is introduced, which provides full transmit-diversity equal to two.

Contribution: Motivated by the small amount of previous work related to precoding for SM systems, we propose a feedback-based precoding technique, which is shown to boost the performance of these systems, both in terms of diversity and coding gain. More specifically, our work is focused on GSSK modulation for Multiple-Input-Single-Output (MISO) systems. We assume that the phases of the channel links can be made available to the transmitter by exploiting a low-delay feedback link. The MISO setup is here studied for two reasons: i) multiple-active transmit-antennas can be exploited to achieve transmit-diversity; and ii) MISO systems have a high practical importance due to widespread use of one-antenna pocket-sized terminals. In these systems, an efficient way to improve the performance of the mobile units is to exploit advanced signal processing solutions for transmit-diversity. In particular, our goal is to simultaneously achieve high diversity and coding gain. The proposed scheme can be thought as a generalization of the method recently proposed in [12] for only two transmitantennas. We analytically show that for the perfect feedback case our solution offers full transmit-diversity for three and four transmit-antennas without reducing the achievable rate. Furthermore, we take into account the case of imperfect feedback that arises from the quantization of the channel phases for which we assume scalar quantization, where each channel phase is quantized seperately. Numerical examples show that for various MISO configurations and achievable rates up to $4 \mathrm{bits} / \mathrm{s} / \mathrm{Hz}$ our proposed method achieves a performance significantly better than state-of-the-art open-loop GSSK modulation schemes when between 2 and 4 bits are allocated for the quantization of each channel phase.

Organization: This paper is organized as follows. In Section II the system model is introduced, and state-of-the-art GSSK and TOSD-GSSK modulations are summarized. In Section III, the proposed adaptive GSSK method is described. In Section IV, Monte Carlo simulations are shown to substantiate our claims, and highlight the performance gain against state-ofthe-art methods. Finally, Section V concludes this paper.

\section{System Model AND BACKGROUND}

\section{A. System Model}

We consider a generic MISO communication system with $N_{t}$ transmit antennas and $N_{r}=1$ receive antennas. We assume that the receiver uses a Maximum-Likelihood (ML) detector [13], and that it is able to estimate the $N_{t}$ channel impulse responses perfectly. Consequently, the receiver has perfect CSI. Furthermore, an error-free and zero-delay feedback channel between receiver and transmitter is assumed to be available. We consider that the $N_{t} \times 1$ MISO channel matrix $\mathbf{H}$ has entries $h_{i}, i=1,2, \ldots N_{t}$, which can be modeled as independent and identically distributed complex Gaussian random variables with zero-mean and unit-variance (Rayleigh fading).

Notation: The following notation is used throughout this paper: i) $N_{a}$ is the number of simultaneously active transmitantennas, with $1 \leq N_{a} \leq N_{t}$; ii) $E_{m}$ is the average total energy transmitted at each time slot by the $N_{\alpha}$ active transmitantennas. An equal distribution among the active transmitantennas is assumed, i.e. each active antenna emits a signal with energy $E_{m} / N_{a}$; iii) $\eta$ is the Additive White Gaussian Noise (AWGN) at the receiver input, with both real and imaginary parts having a power spectral density (PSD) equal to $N_{0} / 2$; iv) $Q(x)=(1 / \sqrt{2 \pi}) \int_{x}^{\infty} \exp \left(-u^{2} / 2\right) d u$ denotes the Q-function; v) $\bar{\gamma}=E_{m} / 2 N_{0}$; vii) $\operatorname{comb}(n, r)=n ! /(r !(n-$ $r)$ !), with $r \leq n$; vi) $\lfloor\cdot\rfloor$ denotes the floor function of a real number; and vii) vectors are denoted in boldface.

\section{B. GSSK Modulation}

In GSSK modulation [4], the source generates a random sequence of independent bits, which are grouped in sets of $m=\left\lfloor\log _{2}\left[\operatorname{comb}\left(N_{t}, N_{a}\right)\right]\right\rfloor$ and mapped to one of the possible $N_{H}=2^{m}$ points of the spatial-constellation diagram. If the $j-$ th spatial-constellation point is chosen, with $j=1,2, \ldots, N_{H}$, then the received signal at a given time-slot is given by:

$$
\left.y\right|_{j}=\left(\begin{array}{llll}
h_{j(1)} s_{j(1)} & h_{j(2)} s_{j(2)} & \cdots & h_{j\left(N_{\alpha}\right)} s_{\left.j N_{\alpha}\right)}
\end{array}\right) \mathbf{w}_{j}
$$

where $j(l) \in\left\{1,2, \ldots, N_{t}\right\}, l=1,2, \ldots, N_{a}$, denotes the index of the $l-t h$ antenna in the $j-t h$ spatial-constellation point. $s_{j(l)}$ denotes the transmitted symbol of the $j(l)-t h$ antenna. For GSSK modulation, $s_{j(l)}(t)=\sqrt{E_{m}} u(t) \forall j(l) \in$ $\left\{1,2, \ldots, N_{t}\right\}$ where $u(\cdot)$ denotes the unit-energy pulse waveform for each transmission, i.e., $\int_{-\infty}^{\infty}|u(t)|^{2} d t=1 . \mathbf{w}_{j}$ is a (complex) $N_{a} \times 1$ vector containing the transmit weights of the $N_{a}$ antennas that belong to the $j-t h$ spatial-constellation point, under the energy constraint $\left\|\mathbf{w}_{j}\right\|^{2} \leq 1$. In GSSK modulation [4], no CSIT is assumed. Thus, the transmit weights are real numbers, which are designed to provide equal energy allocation, i.e., $w_{j i}=1 / \sqrt{N_{a}}$, for $j=1,2, \ldots, N_{H}$ and $i=1,2, \ldots, N_{a}$. During the detection process, the MLoptimum receiver solves a $N_{H}$-hypothesis testing problem for the estimation of the index of the $N_{a} a$ active antennas. This way, the unique message emitted by the encoder is detected. The achievable rate of GSSK modulation is equal to $R_{G S S K}=\left\lfloor\log _{2}\left[\operatorname{comb}\left(N_{t}, N_{a}\right)\right]\right\rfloor$.

The Bit Error Probability (BEP) of GSSK modulation has been computed in [4] and [15] for the ML-optimum receiver. In particular, by using [15, Eq. (35)], we have:

$$
\mathrm{BEP}_{G S S K} \leq A \sum_{j}^{N_{H}} \sum_{k, k \neq j}^{N_{H}} N_{b_{j, k}} Q\left(\sqrt{\frac{\bar{\gamma}}{N_{a}}\left|\sum_{l=1}^{N_{a}}\left[h_{j(l)}-h_{k(l)}\right]\right|^{2}}\right)
$$

where $A=1 /\left[N_{H} \log _{2}\left(N_{H}\right)\right]$ and $N_{b_{j, k}}$ denotes the number of bits in error between the $j-t h$ and $k-t h$ spatial-constellation points, which depends on the GSSK mapper rule [4]. These symbols will also be used in the rest of this paper. From (2), it is evident that no transmit-diversity can be achieved 
due to the coherent summation of complex Gaussian channel gains. Furthermore, energy is wasted due to the subtraction of the channel gains of common antennas in different spatialconstellation points.

\section{TOSD-GSSK Modulation}

Unlike GSSK modulation, TOSD-GSSK modulation can achieve transmit-diversity by transmitting time-orthogonal signals from the active transmit-antennas [5, Eq. (21)], [6]. Similar to GSSK modulation, the entries $w_{j i}$ for $j=1,2, \ldots, N_{H}$ and $i=1,2, \ldots, N_{a}$ of the precoding vector $\mathbf{w}_{j}$ are equal to $1 / \sqrt{N_{a}}$, due to the lack of CSIT. The BEP of TOSD-GSSK can be computed as follows [6]:

$$
\mathrm{BEP}_{T O S D-G S S K} \leq A \sum_{j}^{N_{H}} \sum_{k, k \neq j}^{N_{H}} N_{b_{j, k}} Q\left(\sqrt{\frac{\bar{\gamma}}{N_{a}}\left\|h_{j, q}\right\|^{2}}\right)
$$

where $h_{j, q}$ is a vector that contains all the distinct channel gains between the spatial-constellation points $j$ and $k$. From (3), it follows that even though TOSD-GSSK achieves transmit-diversity by using orthogonal waveforms, it does not solve the problem of wasting energy due to the elimination of common channel links among spatial-constellation points.

\section{Adaptive GSSK Modulation}

In this section, we introduce a new GSSK modulation scheme, which exploits precoding and CSIT at the transmitter to improve diversity and coding gain. To make the precoding design simpler, we exploit only the knowledge of the channel phases, which are assumed to be available at the transmitter through a feedback channel in the case of Frequency-Division Duplexing (FDD) systems. By setting the phase of one of the $N_{t}$ wireless links as a reference, denoted as $\phi_{r e f}$, the receiver needs to feedback to the transmitter only $N_{t}-1$ phase differences. We denote the phases of the $N_{t}$ channel links by $\phi_{i}, i=1,2, \ldots, N_{t}$. If, for instance, $\varphi_{\text {ref }}=\varphi_{1}$, the feedback vector $\phi_{\text {feedback }}$ of the phase differences is given by: $\phi_{\text {feedback }}=\left(\begin{array}{lll}\phi_{1}-\phi_{2} & \cdots & \phi_{1}-\phi_{N_{t}}\end{array}\right)$. Similar to GSSK modulation, no orthogonal waveforms are used.

\section{A. Design of Precoding Vectors}

The design of the precoding vectors $\mathbf{w}_{j}, j=1,2, \ldots, N_{H}$ is based on the following observation. From (2), it follows that the main reason why SSK and GSSK modulations cannot achieve transmit-diversity is due to the coherent summation of the channel gains, which is caused by the presence of random channel phases in the spatial-constellation points. The main idea of the proposed precoding design is to avoid random channel phases. To this end, our method is based on cophasing and phase-rotation operations. In particular: i) for any spatial-constellation point, the channel phases of the $N_{a}$ active antennas are aligned to $\phi_{\text {ref }}$, i.e., they are co-phased. This step is equivalent to conventional Equal Gain Combining (EGC) [16] at the receiver, but it is performed at the transmitted by exploiting the knowledge of $\phi_{\text {feedback }}$; and ii) the resulting cophased $N_{H}$ spatial-constellation points are adequately phaserotated in order to maximize the Euclidean distance among all of them. This step is equivalent to uniformly distributing the co-phased spatial-constellation points over a circle, similar to Phase Shift Keying (PSK) modulation [13]. As a result, the spatial-constellation diagram resulting after applying the precoding vector would geometrically resemble a PSK modulation scheme, whose $N_{H}$ points are random variables given by the summation of $N_{a}$ independent Rayleigh channel gains. Accordingly, the $j-t h$ spatial-constellation point, after being phase-aligned, is phase-rotated by $(j-1)\left(2 \pi / N_{H}\right)$ for $j=1,2, \ldots N_{H}$, which is the same for each time-slot.

In summary, the $j-t h$ spatial-constellation point after applying precoding at the transmitter is:

$$
x_{j}=\sqrt{E_{m}}\left(\left|h_{j(1)}\right| e^{j \phi_{j(1)}} \cdots \cdots \quad\left|h_{j\left(N_{a}\right)}\right| e^{j \phi_{j\left(N_{a}\right)}}\right) \mathbf{w}_{j}
$$

where $h_{j(l)}=\left|h_{j(l)}\right| e^{j \phi_{j(l)}}$ for $l=1,2, \ldots, N_{a}$, and the precoding vector $\mathbf{w}_{j}$, which takes into account co-phasing and rotation, is:

$$
\mathbf{w}_{j}=\frac{1}{\sqrt{N_{a}}}\left(\begin{array}{c}
e^{j\left(\varphi_{r e f}+(j-1) \frac{2 \pi}{N_{H}}-\varphi_{j(1)}\right)} \\
\vdots \\
e^{j\left(\varphi_{r e f}+(j-1) \frac{2 \pi}{N_{H}}-\varphi_{j\left(N_{a}\right)}\right)}
\end{array}\right)
$$

Consequently, (4) simplifies as follows:

$$
x_{j}=\sqrt{\frac{E_{m}}{N_{a}}}\left(\sum_{l=1}^{N_{a}}\left|h_{j(l)}\right|\right) e^{j\left(\varphi_{r e f}+(j-1) \frac{2 \pi}{N_{H}}\right)}
$$

for $j=1,2, \ldots N_{H}$, which resembles an $N_{H}$-PSK constellation diagram with points being random variables that depend on the channel gains.

\section{B. Performance Analysis}

By using again [15, Eq. (35)], the BEP of the proposed adaptive method can be computed as follows:

$$
\mathrm{BEP}_{\text {Adaptive-GSSK }} \leq A \sum_{j}^{N_{H}} \sum_{k, k \neq j}^{N_{H}} N_{b_{j, k}} P E P_{x_{j} \rightarrow x_{k} \mid h}
$$

where $P E P_{x_{j} \rightarrow x_{k} \mid h}$ is the Pairwise Error Probability (PEP) between the spatial-constellation points $x_{j}$ and $x_{k}$ in (6), conditioned on the fading paths. This PEP can be computed as follows:

$$
\begin{aligned}
P E P_{x_{j} \rightarrow x_{k} \mid h} & =Q\left(\sqrt{\frac{1}{2 N_{0}}\left|x_{j}-x_{k}\right|^{2}}\right) \\
& =Q\left(\sqrt{\frac{1}{2 N_{0}}\left[\left|x_{j}\right|^{2}+\left|x_{k}\right|^{2}-2\left|x_{j}\right|\left|x_{k}\right| \cos \left(\phi_{j, k}\right)\right]}\right)
\end{aligned}
$$

where $\varphi_{j, k}=|j-k|\left(2 \pi / N_{H}\right) \in(0, \pi]$ is the phase difference between $x_{j}$ and $x_{k}$, which, unlike (2), is no longer a random variable.

By comparing (8) with (2) and (3), we notice that the fading gains between pairs of spatial-constellation points do not cancel out, and that no random phases are present in the expression of the PEP. The benefit is twofold: i) high coding gain; and ii) high diversity gain. In the next section, we study 
analytically the achievable diversity order of the proposed modulation scheme when three and four antennas are used at the transmitter. The analysis of system setups with a larger number of transmit-antennas is postponed to future research.

\section{Analysis of the Diversity Order}

Three Transmit-Antennas: Let us consider a setup with $N_{t}=$ 3 and $N_{a}=2$, where $N_{H}=2$ and $R=1$ bits $/ \mathrm{s} / \mathrm{Hz}$. Without loss of generality, we assume that the spatial-constellation diagram is composed by the pairs of antenna indexes given by $(1,2)$ and $(1,3)$, which correspond to the transmission of bits ' 0 ' and '1', respectively, and $\varphi_{r e f}=\varphi_{1}$. By using (4) and (5), we have:

$$
\begin{aligned}
x_{1} & =\sqrt{E_{m}}\left(\left|h_{1}\right| e^{j \phi_{1}}\left|h_{2}\right| e^{j \phi_{2}}\right)\left(\begin{array}{l}
1 / \sqrt{N_{a}} \\
e^{j\left(\phi_{1}-\phi_{2}\right)} / \sqrt{N_{a}}
\end{array}\right) \\
& =\sqrt{\frac{E_{m}}{N_{a}}}\left(\left|h_{1}\right|+\left|h_{2}\right|\right) e^{j \phi_{1}}
\end{aligned}
$$

and

$$
\begin{aligned}
x_{2} & =\sqrt{E_{m}}\left(\left|h_{1}\right| e^{j \phi_{1}}\left|h_{3}\right| e^{j \phi_{3}}\right)\left(\begin{array}{l}
e^{j \pi} / \sqrt{N_{a}} \\
e^{j\left(\phi_{1}-\phi_{3}+\pi\right)} / \sqrt{N_{a}}
\end{array}\right) \\
& =\sqrt{\frac{E_{m}}{N_{a}}}\left(\left|h_{1}\right|+\left|h_{3}\right|\right) e^{j\left(\phi_{1}+\pi\right)}
\end{aligned}
$$

By substituting (9) and (10) in (8), we obtain:

$$
\begin{aligned}
P E P_{0^{\prime} 0^{\prime} 1^{\prime} \mid h} & =P E P^{\prime} 1^{\prime} \rightarrow^{\prime} 0^{\prime} \mid h \\
& =Q\left(\sqrt{\frac{\bar{\gamma}}{N_{a}}}\left[2\left|h_{1}\right|+\left|h_{2}\right|+\left|h_{3}\right|\right]\right)
\end{aligned}
$$

By carefully analyzing (11), we notice that is resembles, except for a scaling factor, the PEP of a system with EGC and three receive-antennas [16]. Thus, we can conclude that a diversity order equal to $L=N_{t}=3$ is obtained.

Four Transmit-Antennas: If $N_{t}=4$, the angle $\phi_{j, k}$ between a pair $\left(x_{j}, x_{k}\right)$ of spatial-constellation points can take two different values: i) $\phi_{j, k}=\pi$; and ii) $\phi_{j, k}=\pi / 2$.

Case $\phi_{j, k}=\pi$ : Using (6) and (8), we obtain:

$$
\begin{aligned}
P E P_{x_{j} \rightarrow x_{k} \mid h} & =Q\left(\sqrt{\frac{\bar{\gamma}}{N_{a}}}\left[\sum_{l=1}^{N_{a}}\left[\left|h_{j(l)}\right|+\left|h_{k(l)}\right|\right]\right]\right) \\
& =Q\left(\sqrt{\frac{\bar{\gamma}}{N_{a}}}\left[\sum_{i}^{2 d_{j, k}}\left|h_{i}\right|+\sum_{q, q \neq i}^{N_{a}-d_{j, k}} 2\left|h_{q}\right|\right]\right)
\end{aligned}
$$

where $2 d_{j, k}$ is the number of distinct antenna-indexes between the spatial-constellation points $x_{j}$ and $x_{k}$. Once again, (12) resembles an EGC diversity scheme with $L_{j, k}=2 d_{j, k}+N_{a}$ $d_{j, k}=N_{a}+d_{j, k}$ antennas at the receiver. Thus, the diversity gain of (12) is $L_{j, k}$.

Case $\phi_{j, k}=\pi / 2$ : By still using (6) and (8), we obtain:

$P E P_{x_{j} \rightarrow x_{k} \mid h}=Q\left(\sqrt{\frac{\bar{\gamma}}{N_{a}}\left[\sum_{i}^{2 d_{j, k}}\left|h_{i}\right|^{2}+\sum_{q, q \neq i}^{N_{a}-d_{j, k}} 2\left|h_{q}\right|^{2}+f(h)\right]}\right)$ where $f(h)$ is given by the summation of the mixed products of Rayleigh random variables. Thus, $f(h) \geq 0$ for every channel realization. The diversity order $L_{j, k}$ of (13) can be obtained by using the following upper-bound:

$$
P E P_{x_{j} \rightarrow x_{k} \mid h} \leq Q\left(\sqrt{\frac{\bar{\gamma}}{N_{a}}\left[\sum_{i}^{2 d_{j, k}}\left|h_{i}\right|^{2}+\sum_{q, q \neq i}^{N_{a}-d_{j, k}} 2\left|h_{q}\right|^{2}\right]}\right)
$$

which stems from the fact that $f(h) \geq 0$ and that the Qfunction is monotonically decreasing for increasing values of its argument. By carefully studying (14), we can notice that the PEP resembles a Maximum Ratio Combining (MRC) diversity scheme [16] with $L_{j, k}=2 d_{j, k}+N_{a}-d_{j, k}=N_{a}+d_{j, k}$ antennas at the receiver. Thus, the diversity order of (13) is $L_{j, k} \geq N_{a}+d_{j, k}$. If $N_{t}=4$, we conclude that different PEPs might have different diversity gains. Overall, the diversity order $L$ of the system is equal to the minimum of the diversity orders of the PEPs, i.e., $L=\min \left\{L_{j, k}\right\}$ for $j, k=1,2, \ldots, N_{H}$.

Full-Diversity Order $-N_{a}=3$. Let us consider the setup with $N_{a}=3$. There are in total four combinations of three simultaneously active antennas, i.e., $(1,2,3),(1,2,4),(2,3,4)$, and $(1,3,4)$. Thus, $N_{H}=4$. By direct inspection, we have $2 d_{j, k}=$ 2 for $j, k=1,2, \ldots, N_{H}$. So, $\left.L\right|_{\varphi_{j, k=\pi}}=N_{a}+d_{j, k}=4$ and $\left.L\right|_{\varphi_{j, k=\pi / 2}} \geq N_{a}+d_{j, k}=4$. Consequently, $L=N_{t}=4$. Since there are four constellation points, the achievable rate is equal to $R=\log _{2}(4)=2 \mathrm{bits} / \mathrm{s} / \mathrm{Hz}$, which is the maximum achievable rate for $N_{t}=4$.

\section{NumericAl RESUlts}

The aim of this section is to show some numerical examples to validate the claims made in Section III regarding the performance of the proposed GSSK modulation scheme with precoding. The analysis is conducted by using Monte Carlo simulations and (7) for four different scenarios, as seen in Fig.1. Furthermore, we compare the performance of the proposed scheme with state-of-the-art GSSK and TOSDGSSK modulations. For the bit mapping of (7), we used Gray coding.

We observe a very close agreement between Monte Carlo simulations and the analytical framework in (7), especially in the high-SNR region. Also, the results confirm our claims regarding the coding and diversity gain advantage of our proposed method against other solutions available in the literature in the ideal case of a perfect feedback of the channel phases. For the practical case of limited feedback, since the channel phases are uniformly distributed at $[-\pi, \pi]$ we considered scalar uniform quantization where $B$ bits are allocated for the quantization of each channel phase. Consequently, $-\pi+\left(2 \pi k / 2^{B}\right), k=1,2, \ldots, 2^{B}$, are the $2^{B}$ quantization levels and $B\left(N_{t}-1\right)$ is the total number of bits required for the feedback information. As we see in Fig.1, for a rate up to $3 \mathrm{bits} / \mathrm{s} / \mathrm{Hz}$, just 2 bits per channel phase is an adequate number to achieve a significantly better performance than the state-of-the-art GSSK schemes. For scenario d) where the 

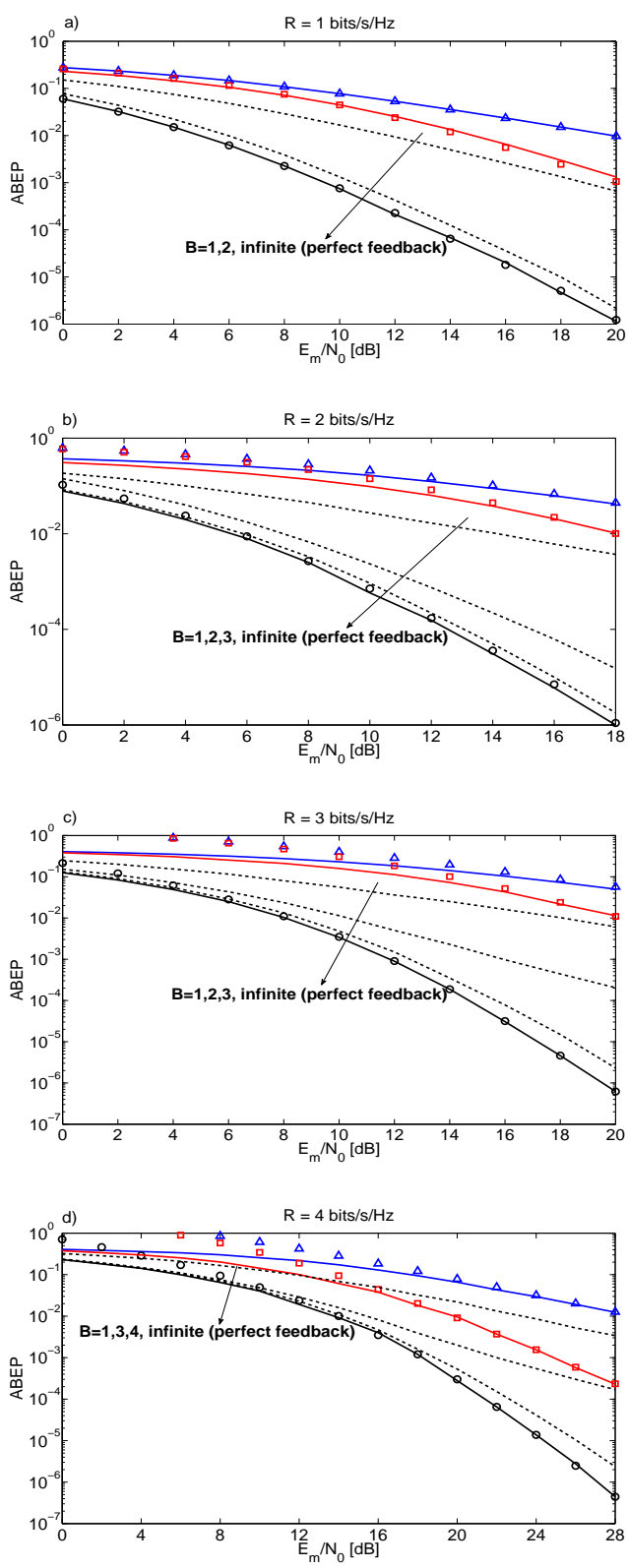

Fig. 1. Average (over fading channels) BEP vs. $E_{m} / N_{0}$ for: a) $N_{t}=$ $3, N_{a}=2$, b) $N_{t}=4, N_{a}=3$, c) $N_{t}=6, N_{a}=4$, and d) $N_{t}=6, N_{a}=3$. Solid lines show Monte Carlo simulations and markers show the analytical model: GSSK $\rightarrow \Delta$, TOSD-GSSK $\rightarrow \square$, Adaptive-GSSK $\rightarrow \bigcirc$. The dashed lines show the Monte Carlo simulations for the limited feedback case. $B$ is the number of feedback bits per channel phase.

achievable rate is $4 \mathrm{bits} / \mathrm{s} / \mathrm{Hz}$, we see that 4 bits of feedback per chanel phase are needed to significantly outperform these GSSK schemes. Such a higher number of feedback bits is intuitively expected due to the higher number of constellation points.

\section{CONCLUSions AND Future Work}

In this paper, we have proposed a new precoding method for the optimization of the spatial-constellation diagram of GSSK modulation. The method exploits co-phasing and phaserotation to achieve better diversity and coding gains. We have analytically shown that a full-diversity gain can be achieved for three and four transmit-antennas without reducing the achievable rate for the ideal case of a perfect feedback. In the practical case of limited feedback, we have shown through Monte Carlo simulations that between 2 and 4 bits of feedback per channel phase is an adequate number to significantly outperform the state-of-the-art GSSK modulation schemes for various rates. This makes our proposed method suitable for applications in slowly fading channels where the feedback information is not sent at each time slot, which reduces the feedback overhead.

Future research is concerned with the mathematical analysis of the impact of quantized CSIT on the achievable transmitdiversity. Vector quantization will be also considered besides scalar quantization. Furthermore, the analysis of system setups with multiple receive-antennas and the design of precoders robust to CSI estimation errors are also topics of interest.

\section{REFERENCES}

[1] R. Y. Mesleh et al., "Spatial modulation", IEEE Trans. Veh. Technol., vol. 57, no. 4, pp. 2228-2241, July 2008.

[2] J. Jeganathan, A. Ghrayeb, L. Szczecinski, "Spatial modulation: Optimal detection and performance analysis", IEEE Commun. Lett., vol. 12, pp. 545-547, Aug. 2008.

[3] J. Jeganathan et al., "Space shift keying modulation for MIMO channels", IEEE Trans. Wireless Commun., vol. 8, no. 7, pp. 3692-3703, July 2009.

[4] J. Jeganathan, A. Ghrayeb, and L. Szczecinski, "Generalized space shift keying modulation for MIMO channels", IEEE PIMRC, pp. 1-5, Sep. 2008.

[5] M. Di Renzo and H. Haas, "SSK-MIMO over correlated Rician fading channels: Performance analysis and a new method to achieve transmitdiversity gains", IEEE Trans. Commun., vol. 59, no. 1, pp. 116-129, Jan 2011.

[6] M. Di Renzo and H. Haas, "Space shift keying (SSK) modulation: On the transmit-diversity/multiplexing trade-off", IEEE ICC, pp. 1-6, June 2011.

[7] M. Di Renzo and H. Haas, "Transmit-diversity for spatial modulation (SM): Towards the design of high-rate spatially-modulated space-time block codes", IEEE ICC, pp. 1-6, June 2011.

[8] E. Basar et al., "Space-time block coded spatial modulation", IEEE Trans. Commun., vol. 59, no.3, pp. 823-832, Mar. 2011.

[9] S. Sugiura, S. Chen, and L. Hanzo, "Coherent and differential space-time shift keying: A dispersion matrix approach", IEEE Trans. Commun., vol. 58, no. 11 , pp. 3219-3230, Nov. 2010.

[10] M. Vu and A. Paulraj, "MIMO wireless linear precoding", IEEE Signal Process. Mag., vol. 24, no. 5, pp. 86-105, Oct. 2007.

[11] T. Handte, A. Muller, and J. Speidel, "BER analysis and optimization of generalized spatial modulation in correlated fading channels", IEEE VTC-Fall, pp. 1-5, Sep. 2009.

[12] D. Yang, L. Yang, and L. Hanzo, "Transmit-diversity-assisted spaceshift keying for co-located and distributed/cooperative MIMO element", IEEE Trans. Veh. Technol., vol. 60, no. 6, pp. 2864-2869, June 2011.

[13] J. Proakis, Digital Communications, 4th ed. New York: McGraw-Hill, August 2000.

[14] M. Di Renzo and H. Haas, "Improving the performance of space shift keying (SSK) modulation via opportunistic power allocation", IEEE Commun. Lett., vol. 14, no. 6, pp. 500-502, June 2010.

[15] M. Di Renzo and H. Haas, "A general framework for performance analysis of space shift keying (SSK) modulation for MISO correlated Nakagami-m fading channels", IEEE Trans. Commun., vol. 58, no. 9, pp. 2590-2603, Sep.2010.

[16] A. Goldsmith, Wireless Communications, Cambridge University Press, August 2005.

[17] V. Tarokh, H. Jafarkhani, and A. R. Calderbank, "Space-time block codes from orthogonal designs", IEEE J. Sel. Areas Commun., vol. 16, pp. 1451-1458, Oct. 1998. 